JOURNAL OF

FUNCTION SPACES AND APPLICATIONS

Volume 7, Number 3 (2009), 301-311 (c) 2009, Scientific Horizon

http://www.jfsa.net

\title{
Remark on the boundedness of the Cauchy singular integral operator on variable Lebesgue spaces with radial oscillating weights
}

\author{
Alexei Yu. Karlovich ${ }^{1}$ \\ To Professor Kokilashvili on his seventieth birthday \\ (Communicated by Lech Maligranda)
}

2000 Mathematics Subject Classification. Primary 42B20; Secondary $47 \mathrm{~B} 38$.

Keywords and phrases. Variable Lebesgue space, Carleson curve, variable exponent, radial oscillating weight, Matuszewska-Orlicz indices, submultiplicative function.

Abstract. Recently V. Kokilashvili, N. Samko, and S. Samko have proved a sufficient condition for the boundedness of the Cauchy singular integral operator on variable Lebesgue spaces with radial oscillating weights over Carleson curves. This condition is formulated in terms of Matuszewska-Orlicz indices of weights. We prove a partial converse of their result.

\section{Introduction and main result}

Let $\Gamma$ be a rectifiable curve in the complex plane. We equip $\Gamma$ with Lebesgue length measure $|d \tau|$. We say that a curve $\Gamma$ is simple if it does not have self-intersections. In other words, $\Gamma$ is said to be simple if it is homeomorphic either to a line segment or to to a circle. In the latter

\footnotetext{
${ }^{1}$ The author is partially supported by F.C.T. (Portugal) grant FCT/FEDER/ POCTI/MAT /59972/2004.
} 
situation we will say that $\Gamma$ is a Jordan curve. The Cauchy singular integral of $f \in L^{1}(\Gamma)$ is defined by

$$
(S f)(t):=\frac{1}{\pi i} \int_{\Gamma} \frac{f(\tau)}{\tau-t} d \tau \quad(t \in \Gamma) .
$$

This integral is understood in the principal value sense, that is,

$$
\int_{\Gamma} \frac{f(\tau)}{\tau-t} d \tau:=\lim _{R \rightarrow 0} \int_{\Gamma \backslash \Gamma(t, R)} \frac{f(\tau)}{\tau-t} d \tau
$$

where $\Gamma(t, R):=\{\tau \in \Gamma:|\tau-t|<R\}$ for $R>0$. David [4] (see also $[3$, Theorem 4.17]) proved that the Cauchy singular integral generates the bounded operator $S$ on the Lebesgue space $L^{p}(\Gamma), 1<p<\infty$, if and only if $\Gamma$ is a Carleson (Ahlfors-David regular) curve, that is,

$$
\sup _{t \in \Gamma} \sup _{R>0} \frac{|\Gamma(t, R)|}{R}<\infty,
$$

where for any measurable set $\Omega \subset \Gamma$ the symbol $|\Omega|$ denotes its measure. To have a better idea about Carleson curves, consider the following example. Let $\alpha>0$ and

$$
\Gamma:=\{0\} \cup\left\{\tau \in \mathbb{C}: \tau=x+i x^{\alpha} \sin (1 / x), 0<x \leq 1\right\} .
$$

One can show (see [3, Example 1.3]) that $\Gamma$ is not rectifiable for $0<\alpha \leq 1$, $\Gamma$ is rectifiable but not Carleson for $1<\alpha<2$, and $\Gamma$ is a Carleson curve for $\alpha \geq 2$.

A measurable function $w: \Gamma \rightarrow[0, \infty]$ is referred to as a weight function or simply a weight if $0<w(\tau)<\infty$ for almost all $\tau \in \Gamma$. Suppose $p: \Gamma \rightarrow[1, \infty]$ is a measurable a.e. finite function. Denote by $L^{p(\cdot)}(\Gamma, w)$ the set of all measurable complex-valued functions $f$ on $\Gamma$ such that

$$
\int_{\Gamma}|f(\tau) w(\tau) / \lambda|^{p(\tau)}|d \tau|<\infty
$$

for some $\lambda=\lambda(f)>0$. This set becomes a Banach space when equipped with the Luxemburg-Nakano norm

$$
\|f\|_{p(\cdot), w}:=\inf \left\{\lambda>0: \int_{\Gamma}|f(\tau) w(\tau) / \lambda|^{p(\tau)}|d \tau| \leq 1\right\}
$$

If $p$ is constant, then $L^{p(\cdot)}(\Gamma, w)$ is nothing else but the weighted Lebesgue space. Therefore, it is natural to refer to $L^{p(\cdot)}(\Gamma, w)$ as a weighted generalized Lebesgue space with variable exponent or simply as a weighted 
variable Lebesgue space. This is a special case of Musielak-Orlicz spaces [19] (see also [13]). Nakano [20] considered these spaces (without weights) as examples of so-called modular spaces, and sometimes the spaces $L^{p(\cdot)}(\Gamma, w)$ are referred to as weighted Nakano spaces.

Following [12, Section 2.3], denote by $W$ the class of all continuous functions $\varrho:[0,|\Gamma|] \rightarrow[0, \infty)$ such that $\varrho(0)=0, \varrho(x)>0$ if $0<x \leq|\Gamma|$, and $\varrho$ is almost increasing, that is, there is a universal constant $C>0$ such that $\varrho(x) \leq C \varrho(y)$ whenever $x \leq y$. Further, let $\mathbb{W}$ be the set of all functions $\varrho:[0,|\Gamma|] \rightarrow[0, \infty]$ such that $x^{\alpha} \varrho(x) \in W$ and $x^{\beta} / \varrho(x) \in W$ for some $\alpha, \beta \in \mathbb{R}$. Clearly, the functions $\varrho(x)=x^{\gamma}$ belong to $\mathbb{W}$ for all $\gamma \in \mathbb{R}$. For $\varrho \in \mathbb{W}$, put

$$
\Phi_{\varrho}^{0}(x):=\limsup _{y \rightarrow 0} \frac{\varrho(x y)}{\varrho(y)}, \quad x \in(0, \infty) .
$$

Since $\varrho \in \mathbb{W}$, one can show that the limits

$$
m(\varrho):=\lim _{x \rightarrow 0} \frac{\log \Phi_{\varrho}^{0}(x)}{\log x}, \quad M(\varrho):=\lim _{x \rightarrow \infty} \frac{\log \Phi_{\varrho}^{0}(x)}{\log x}
$$

exist and $-\infty<m(\varrho) \leq M(\varrho)<+\infty$. These numbers were defined by Matuszewska and Orlicz [17, 18] (see also [15] and [16, Chapter 11]). We refer to $m(\varrho)$ (resp. $M(\varrho)$ ) as the lower (resp. upper) Matuszewska-Orlicz index of $\varrho$. For $\varrho(x)=x^{\gamma}$ one has $m(\varrho)=M(\varrho)=\gamma$. Examples of functions $\varrho \in \mathbb{W}$ with $m(\varrho)<M(\varrho)$ can be found, for instance, in [1], [16, p. 93], [21, Section 2].

Fix pairwise distinct points $t_{1}, \ldots, t_{n} \in \Gamma$ and functions $w_{1}, \ldots, w_{n} \in \mathbb{W}$. Consider the following weight

$$
w(t):=\prod_{k=1}^{n} w_{k}\left(\left|t-t_{k}\right|\right), \quad t \in \Gamma .
$$

Each function $w_{k}\left(\left|t-t_{k}\right|\right)$ is a radial oscillating weight. The weight (1.1) is a continuous function on $\Gamma \backslash\left\{t_{1}, \ldots, t_{n}\right\}$. This is a natural generalization of so-called Khvedelidze weights $w(t)=\prod_{k=1}^{n}\left|t-t_{k}\right|^{\lambda_{k}}$, where $\lambda_{k} \in \mathbb{R}$ (see, e.g., [3, Section 2.2], [9], [10]). Recently V. Kokilashvili, N. Samko, and S. Samko have proved the following (see [12, Theorem 4.3] and also [11] for similar results for maximal functions).

Theorem 1.1 ([12, Theorem 4.3]). Suppose $\Gamma$ is a simple Carleson curve and $p: \Gamma \rightarrow(1, \infty)$ is a continuous function satisfying

$$
|p(\tau)-p(t)| \leq-A_{\Gamma} / \log |\tau-t| \quad \text { whenever } \quad|\tau-t| \leq 1 / 2,
$$


where $A_{\Gamma}$ is a positive constant depending only on $\Gamma$. Let $w_{1}, \ldots, w_{n} \in \mathbb{W}$ and the weight $w$ be given by (1.1). If

$$
0<1 / p\left(t_{k}\right)+m\left(w_{k}\right), \quad 1 / p\left(t_{k}\right)+M\left(w_{k}\right)<1 \quad \text { for all } k \in\{1, \ldots, n\},
$$

then the Cauchy singular integral operator $S$ is bounded on $L^{p(\cdot)}(\Gamma, w)$.

For the weight $w(t)=\prod_{k=1}^{n}\left|t-t_{k}\right|^{\lambda_{k}},(1.3)$ reads as $0<1 / p\left(t_{k}\right)+\lambda_{k}<1$ for all $k \in\{1, \ldots, n\}$. This condition is also necessary for the boundedness of $S$ on the variable Lebesgue space $L^{p(\cdot)}(\Gamma, w)$ with the Khvedelidze weight $w($ see $[10])$.

The author have proved in [8] that for Jordan curves condition (1.3) is necessary for the boundedness of the operator $S$.

Theorem 1.2 ([8, Corollary 4.3]). Suppose $\Gamma$ is a rectifiable Jordan curve and $p: \Gamma \rightarrow(1, \infty)$ is a continuous function satisfying (1.2). Let $w_{1}, \ldots, w_{n} \in \mathbb{W}$ and the weight $w$ be given by (1.1). If the Cauchy singular integral operator $S$ is bounded on $L^{p(\cdot)}(\Gamma, w)$, then $\Gamma$ is a Carleson curve and (1.3) is fulfilled.

The proof of this result given in [8] essentially uses that $\Gamma$ is closed. In this paper we embark on the situation of non-closed curves. Our main result is a partial converse of Theorem 1.1. It follows from our results $[6,8]$ based on further development of ideas from [3, Chap. 1-3].

Theorem 1.3 (Main result). Let $\Gamma$ be a rectifiable curve homeomorphic to a line segment and $p: \Gamma \rightarrow(1, \infty)$ be a continuous function satisfying (1.2). Suppose $w_{1}, \ldots, w_{n} \in \mathbb{W}$ and the weight $w$ is given by (1.1). If the Cauchy singular integral operator $S$ is bounded on $L^{p(\cdot)}(\Gamma, w)$, then $\Gamma$ is a Carleson curve and

$$
0 \leq 1 / p\left(t_{k}\right)+m\left(w_{k}\right), \quad 1 / p\left(t_{k}\right)+M\left(w_{k}\right) \leq 1 \quad \text { for all } k \in\{1, \ldots, n\} .
$$

Moreover, if there exists an $\varepsilon_{0}>0$ such that the Cauchy singular integral operator $S$ is bounded on $L^{p(\cdot)}\left(\Gamma, w^{1+\varepsilon}\right)$ for all $\varepsilon \in\left(-\varepsilon_{0}, \varepsilon_{0}\right)$, then

$$
0<1 / p\left(t_{k}\right)+m\left(w_{k}\right), \quad 1 / p\left(t_{k}\right)+M\left(w_{k}\right)<1 \quad \text { for all } k \in\{1, \ldots, n\} .
$$

For standard Lebesgue spaces, the boundedness of the operator $S$ on $L^{p}(\Gamma, w), 1<p<\infty$, implies that $S$ is also bounded on $L^{p}\left(\Gamma, w^{1+\varepsilon}\right)$ for all $\varepsilon$ in a sufficiently small neighborhood of zero (see [3, Theorems 2.31 and $4.15])$. Hence if $1<p<\infty, \Gamma$ is a simple Carleson curve, $w_{1}, \ldots, w_{n} \in \mathbb{W}$, and the weight $w$ is given by (1.1), then $S$ is bounded on the standard Lebesgue space $L^{p}(\Gamma, w), 1<p<\infty$, if and only if

$$
0<1 / p+m\left(w_{k}\right), \quad 1 / p+M\left(w_{k}\right)<1 \quad \text { for all } \quad k \in\{1, \ldots, n\} .
$$


We believe that all weighted variable Lebesgue spaces have this stability property.

Conjecture 1.4. Let $\Gamma$ be a simple rectifiable curve, $p: \Gamma \rightarrow[1, \infty]$ be a measurable a.e. finite function, and $w: \Gamma \rightarrow[0, \infty]$ be a weight such that the Cauchy singular integral operator $S$ is bounded on $L^{p(\cdot)}(\Gamma, w)$. Then there is a number $\varepsilon_{0}>0$ such that $S$ is bounded on $L^{p(\cdot)}\left(\Gamma, w^{1+\varepsilon}\right)$ for all $\varepsilon \in\left(-\varepsilon_{0}, \varepsilon_{0}\right)$.

If this conjecture would be true, we were able to prove the complete converse of Theorem 1.1 for non-closed curves, too.

\section{Proof}

In this section we formulate several results from $[3,6,8]$ and show that Theorem 1.3 easily follows from them.

2.1 Muckenhoupt type condition. Suppose $\Gamma$ is a simple rectifiable curve and $p: \Gamma \rightarrow(1, \infty)$ is a continuous function. Since $\Gamma$ is compact, one has

$$
1<\min _{\tau \in \Gamma} p(\tau), \quad \max _{\tau \in \Gamma} p(\tau)<\infty
$$

and the conjugate exponent

$$
q(\tau):=p(\tau) /(p(\tau)-1) \quad(\tau \in \Gamma)
$$

is well defined and also bounded and bounded away from zero. We say that a weight $w: \Gamma \rightarrow[0, \infty]$ belongs to $A_{p(\cdot)}(\Gamma)$ if

$$
\sup _{t \in \Gamma} \sup _{R>0} \frac{1}{R}\left\|w \chi_{\Gamma(t, R)}\right\|_{p(\cdot)}\left\|w^{-1} \chi_{\Gamma(t, R)}\right\|_{q(\cdot)}<\infty .
$$

If $p=$ const $\in(1, \infty)$, then this class coincides with the well known Muckenhoupt class. From the Hölder inequality for $L^{p(\cdot)}(\Gamma)$ (see e.g. [19, Theorems 13.12 and 13.13] for Muslielak-Orlicz spaces over arbitrary measure spaces and also [13, Theorem 2.1] for variable Lebesgue spaces over domains in $\left.\mathbb{R}^{n}\right)$ it follows that if $w \in A_{p(\cdot)}(\Gamma)$, then $\Gamma$ is a Carleson curve.

Since $L^{p(\cdot)}(\Gamma, w)$ is a Banach function space in the sense of $[2$, Definition 1.1], the next result follows from [6, Theorem 6.1] (stated in [6] for Jordan curves, however its proof remains the same for curves homeomorphic to line segments, see also [7, Theorem 3.2]).

Theorem 2.1. Let $\Gamma$ be a simple rectifiable curve and let $p: \Gamma \rightarrow(1, \infty)$ be a continuous function. If $w: \Gamma \rightarrow[0, \infty]$ is an arbitrary weight such that the operator $S$ is bounded on $L^{p(\cdot)}(\Gamma, w)$, then $w \in A_{p(\cdot)}(\Gamma)$. 
If $p=$ const $\in(1, \infty)$, then $w \in A_{p}(\Gamma)$ is also sufficient for the boundedness of $S$ on the weighted Lebesgue space $L^{p}(\Gamma, w)$ (see e.g. [3, Theorem 4.15]).

2.2 Submultiplicative functions. Following [3, Section 1.4], we say a function $\Phi:(0, \infty) \rightarrow(0, \infty]$ is regular if it is bounded in an open neighborhood of 1 . A function $\Phi:(0, \infty) \rightarrow(0, \infty]$ is said to be submultiplicative if

$$
\Phi(x y) \leq \Phi(x) \Phi(y) \quad \text { for all } \quad x, y \in(0, \infty) .
$$

It is easy to show that if $\Phi$ is regular and submultiplicative, then $\Phi$ is bounded away from zero in some open neighborhood of 1 . Moreover, in this case $\Phi(x)$ is finite for all $x \in(0, \infty)$. Given a regular and submultiplicative function $\Phi:(0, \infty) \rightarrow(0, \infty)$, one defines

$$
\alpha(\Phi):=\sup _{x \in(0,1)} \frac{\log \Phi(x)}{\log x}, \quad \beta(\Phi):=\inf _{x \in(1, \infty)} \frac{\log \Phi(x)}{\log x} .
$$

Clearly, $-\infty<\alpha(\Phi)$ and $\beta(\Phi)<\infty$.

Theorem 2.2 (see [3, Theorem 1.13] or [14, Chap. 2, Theorem 1.3]). If a function $\Phi:(0, \infty) \rightarrow(0, \infty)$ is regular and submultiplicative, then

$$
\alpha(\Phi)=\lim _{x \rightarrow 0} \frac{\log \Phi(x)}{\log x}, \quad \beta(\Phi)=\lim _{x \rightarrow \infty} \frac{\log \Phi(x)}{\log x}
$$

and $-\infty<\alpha(\Phi) \leq \beta(\Phi)<+\infty$.

The quantities $\alpha(\Phi)$ and $\beta(\Phi)$ are called the lower and upper indices of the regular and submultiplicative function $\Phi$, respectively.

2.3 Indices of powerlikeness. Fix $t \in \Gamma$ and put $d_{t}:=\max _{\tau \in \Gamma}|\tau-t|$. Suppose $w: \Gamma \rightarrow[0, \infty]$ is a weight such that $\log w \in L^{1}(\Gamma(t, R))$ for every $R \in\left(0, d_{t}\right]$. Put

$$
H_{w, t}\left(R_{1}, R_{2}\right):=\frac{\exp \left(\frac{1}{\left|\Gamma\left(t, R_{1}\right)\right|} \int_{\Gamma\left(t, R_{1}\right)} \log w(\tau)|d \tau|\right)}{\exp \left(\frac{1}{\left|\Gamma\left(t, R_{2}\right)\right|} \int_{\Gamma\left(t, R_{2}\right)} \log w(\tau)|d \tau|\right)}, \quad R_{1}, R_{2} \in\left(0, d_{t}\right]
$$

Consider the function

$$
\left(V_{t}^{0} w\right)(x):=\limsup _{R \rightarrow 0} H_{w, t}(x R, R), \quad x \in(0, \infty) .
$$


Combining Lemmas 4.8-4.9 and Theorem 5.9 of [6] with Theorem 3.4, Lemma 3.5 of [3], we arrive at the following.

Theorem 2.3. Let $\Gamma$ be a simple rectifiable curve, $p: \Gamma \rightarrow(1, \infty)$ be a continuous function satisfying (1.2), and $w: \Gamma \rightarrow[0, \infty]$ be a weight such that $w \in A_{p(\cdot)}(\Gamma)$. Then, for every $t \in \Gamma$, the function $V_{t}^{0} w$ is regular and submultiplicative and

$$
0 \leq 1 / p(t)+\alpha\left(V_{t}^{0} w\right), \quad 1 / p(t)+\beta\left(V_{t}^{0} w\right) \leq 1 .
$$

The numbers $\alpha\left(V_{t}^{0} w\right)$ and $\beta\left(V_{t}^{0} w\right)$ are called the lower and upper indices of powerlikeness of $w$ at $t \in \Gamma$, respectively (see [3, Chap. 3]). This terminology can be explained by the simple fact that for the power weight $w(\tau):=|\tau-t|^{\lambda}$ its indices of powerlikeness coincide and are equal to $\lambda$.

2.4 Matuszewska-Orlicz indices as indices of powerlikeness. If $\varrho \in \mathbb{W}$, then $\Phi_{\varrho}^{0}$ is a regular and submultiplicative function and its indices are nothing else but the Matuszewska-Orlicz indices $m(\varrho)$ and $M(\varrho)$. The next result shows that for radial oscillating weights indices of powerlikeness and Matuszewska-Orlicz indices coincide.

Theorem 2.4 (see [8, Theorem 2.8]). Suppose $\Gamma$ is a simple Carleson curve. If $w_{1}, \ldots, w_{n} \in \mathbb{W}$ and $w(\tau)=\prod_{k=1}^{n} w_{k}\left(\left|\tau-t_{k}\right|\right)$, then for every $t \in \Gamma$ the function $V_{t}^{0} w$ is regular and submultiplicative and

$$
\begin{aligned}
& \alpha\left(V_{t_{k}}^{0} w\right)=m\left(w_{k}\right), \quad \beta\left(V_{t_{k}}^{0} w\right)=M\left(w_{k}\right) \quad \text { for } \quad k \in\{1, \ldots, n\}, \\
& \alpha\left(V_{t}^{0} w\right)=0, \quad \beta\left(V_{t}^{0} w\right)=0 \quad \text { for } t \in \Gamma \backslash\left\{t_{1}, \ldots, t_{n}\right\} .
\end{aligned}
$$

Note that in [8], Theorem 2.4 is proved for Jordan curves. But the proof does not use the assumption that $\Gamma$ is closed. It works also for non-closed curves considered in this paper.

2.5 Proof of Theorem 1.3. Suppose $S$ is bounded on $L^{p(\cdot)}(\Gamma, w)$. From Theorem 2.1 it follows that $w \in A_{p(\cdot)}(\Gamma)$. By Hölder's inequality this implies that $\Gamma$ is a Carleson curve. Fix an arbitrary $t \in \Gamma$. Then, in view of Theorems 2.2 and 2.3 the function $V_{t}^{0} w$ is regular and submultiplicative, so its indices are well defined and satisfy $0 \leq 1 / p(t)+\alpha\left(V_{t}^{0} w\right)$ and $1 / p(t)+\beta\left(V_{t} w\right) \leq 1$. From these inequalities and Theorem 2.4 it follows that

$$
0 \leq 1 / p\left(t_{k}\right)+m\left(w_{k}\right), \quad 1 / p\left(t_{k}\right)+M\left(w_{k}\right) \leq 1
$$

for all $k \in\{1, \ldots, n\}$. 
If $S$ is bounded on all spaces $L^{p(\cdot)}\left(\Gamma, w^{1+\varepsilon}\right)$ for all $\varepsilon$ in a neighborhood of zero, then as before

$$
0 \leq 1 / p\left(t_{k}\right)+m\left(w_{k}^{1+\varepsilon}\right), \quad 1 / p\left(t_{k}\right)+M\left(w_{k}^{1+\varepsilon}\right) \leq 1
$$

for every $k \in\{1, \ldots, n\}$. It is easy to see that $m\left(w_{k}^{1+\varepsilon}\right)=(1+\varepsilon) m\left(w_{k}\right)$ and $M\left(w_{k}^{1+\varepsilon}\right)=(1+\varepsilon) M\left(w_{k}\right)$. Therefore

$$
0 \leq 1 / p\left(t_{k}\right)+(1+\varepsilon) m\left(w_{k}\right), \quad 1 / p\left(t_{k}\right)+(1+\varepsilon) M\left(w_{k}\right) \leq 1
$$

for all $\varepsilon$ in a neighborhood of zero and for all $k \in\{1, \ldots, n\}$. These inequalities immediately imply that $0<1 / p\left(t_{k}\right)+m\left(w_{k}\right)$ and $1 / p\left(t_{k}\right)+$ $M\left(w_{k}\right)<1$ for all $k$.

Remark 2.5. The presented proof involves the notion of indices of powerlikeness, which were invented to treat general Muckenhoupt weights (see [3]). Weights considered in the present paper are continuous except for a finite number of points. So, it would be rather interesting to find a direct proof of the fact that $w \in A_{p(\cdot)}(\Gamma)$ implies (2.1), which does not involve the indices of powerlikeness $\alpha\left(V_{t}^{0} w\right)$ and $\beta\left(V_{t}^{0} w\right)$.

2.6 Final remarks. In connection with Conjecture 1.4, we would like to note that for standard Lebesgue spaces $L^{p}(\Gamma, w)$ there are two different proofs of the stability of the boundedness of $S$ on $L^{p}\left(\Gamma, w^{1+\varepsilon}\right)$ for small $\varepsilon$. Simonenko's proof [22] is based on the stability of the Fredholm property of some singular integral operators related to the Riemann boundary value problem. Another proof is based on the self-improving property of Muckenhoupt weights (see e.g. [3, Theorem 2.31]). One may ask whether does $w \in A_{p(\cdot)}(\Gamma)$ imply $w^{1+\varepsilon} \in A_{p(\cdot)}(\Gamma)$ for all $\varepsilon \in\left(-\varepsilon_{0}, \varepsilon_{0}\right)$ with some fixed $\varepsilon>0$ ? The positive answer would give a proof of the complete converse of (1.3). The author does not know any stability result for the boundedness of $S$ or a self-improving property for $w \in A_{p(\cdot)}(\Gamma)$.

After this paper had been submitted, P. Hästö and L. Diening [5] have found a necessary and sufficient condition for the boundedness of the classical Hardy-Littlewood maximal function on weighted variable Lebesgue spaces in the setting of $\mathbb{R}^{n}$. Note that they write a weight as a measure (outside of $|\cdot|^{p(\tau)}$ ). Their condition is another generalization of the classical Muckenhoupt condition. In the setting of Carleson curves (and the weight written inside of $|\cdot|^{p(\tau)}$ ), the Hästö-Diening condition takes the form

$$
\sup _{t \in \Gamma} \sup _{R>0}\left(\frac{1}{R^{p_{\Gamma(t, R)}}} \int_{\Gamma(t, R)} w(\tau)^{p(\tau)}|d \tau|\right)\left\|w(\cdot)^{-p(\cdot)} \chi_{\Gamma(t, R)}(\cdot)\right\|_{q(\cdot) / p(\cdot)}<\infty,
$$


where

$$
p_{\Gamma(t, R)}:=\left(\frac{1}{|\Gamma(t, R)|} \int_{\Gamma(t, R)} \frac{1}{p(\tau)}|d \tau|\right)^{-1} .
$$

Let $H D_{p(\cdot)}(\Gamma)$ denote the class of weights $w: \Gamma \rightarrow[0, \infty]$ satisfying $(2.2)$. Following the arguments contained in [5, Remark 3.10], one can show that

$$
A_{L^{p(\cdot)}}(\Gamma) \supset H D_{p(\cdot)}(\Gamma)
$$

whenever $p: \Gamma \rightarrow(1, \infty)$ satisfies the Dini-Lipschitz condition (1.2). We conjecture that the Hästö-Diening characterization remains true also for the operator $S$ in the setting of Carleson curves.

Conjecture 2.6. Let $\Gamma$ be a simple Carleson curve, $w: \Gamma \rightarrow[0, \infty]$ be a weight, and $p: \Gamma \rightarrow(1, \infty)$ be a continuous function satisfying the DiniLipschitz condition (1.2). The operator $S$ is bounded on $L^{p(\cdot)}(\Gamma, w)$ if and only if $w \in H D_{p(\cdot)}(\Gamma)$.

Acknowledgement. The author would like to thank the anonymous referee for several useful remarks.

\section{References}

[1] V. D. Aslanov and Yu. I. Karlovich, One-sided invertibility of functional operators in reflexive Orlicz spaces, Akad. Nauk Azerbaidzhan. SSR Dokl., 45 (11-12) (1989), 3-7 (in Russian).

[2] C. Bennett and R. Sharpley, Interpolation of Operators, Academic Press, Boston, 1988.

[3] A. Böttcher and Yu. I. Karlovich, Carleson Curves, Muckenhoupt Weights, and Toeplitz Operators, Birkhäuser, Basel, 1997.

[4] G. David, Operáteurs intégraux singuliers sur certaines courbes du plan complexe, Ann. Sci. École Norm. Super., 17 (1984), 157-189.

[5] P. Hästö and L. Diening, Muckenhoupt weights in variable exponent spaces, preprint.

[6] A. Yu. Karlovich, Fredholmness of singular integral operators with piecewise continuous coefficients on weighted Banach function spaces, J. Integr. Equat. Appl., 15 (2003), 263-320.

[7] A. Yu. Karlovich, Singular integral operators with piecewise continuous coefficients in reflexive rearrangement-invariant spaces, Integral Equatations Operator Theory, 32 (1998), 436-481. 
[8] A. Yu. Karlovich, Singular integral operators on variable Lebesgue spaces with radial oscillating weights, In: "Operator Theory: Advances and Applications", 195 (2010), to appear.

[9] B. V. Khvedelidze, Linear discontinuous boundary problems in the theory of functions, singular integral equations and some of their applications, Trudy Tbiliss. Mat. Inst. Razmadze, 23 (1956), 3-158 (in Russian).

[10] V. Kokilashvili, V. Paatashvili, and S. Samko, Boundedness in Lebesgue spaces with variable exponent of the Cauchy singular operator on Carleson curves, In: "Modern Operator Theory and Applications. The Igor Borisovich Simonenko Anniversary Volume". Operator Theory: Advances and Applications, 170 (2006), 167-186.

[11] V. Kokilashvili, N. Samko, and S. Samko, The maximal operator in weighted variable spaces $L^{p(\cdot)}$, J. Func. Spaces Appl., 5 (2007), 299317.

[12] V. Kokilashvili, N. Samko, and S. Samko, Singular operators in variable spaces $L^{p(\cdot)}(\Omega, \rho)$ with oscillating weights, Math. Nachr., 280 (2007), $1145-1156$.

[13] O. Kováčik and J. Rákosník, On spaces $L^{p(x)}$ and $W^{k, p(x)}$, Czechoslovak Math. J., 41(116) (1991), 592-618.

[14] S. G. Krein, Ju. I. Petunin, and E. M. Semenov, Interpolation of Linear Operators, AMS Translations of Mathematical Monographs, 54, Providence, RI, 1982.

[15] L. Maligranda, Indices and interpolation, Dissert. Math., 234 (1985), $1-49$.

[16] L. Maligranda, Orlicz Spaces and Interpolation, Sem. Math. 5, Dep. Mat., Universidade Estadual de Campinas, Campinas SP, Brazil, 1989.

[17] W. Matuszewska and W. Orlicz, On certain properties of $\varphi$-functions, Bull. Acad. Polon. Sci. Ser. Sci. Math. Astronom. Phys., 8 (1960), 439443. Reprinted in: W. Orlicz, Collected Papers, PWN, Warszawa, 1988, $1112-1116$.

[18] W. Matuszewska and W. Orlicz, On some classes of functions with regard to their orders of growth, Studia Math., 26 (1965), 11-24. Reprinted in: W. Orlicz, Collected Papers, PWN, Warszawa, 1988, 1217-1230.

[19] J. Musielak, Orlicz Spaces and Modular Spaces, Lecture Notes in Mathematics, 1034. Springer-Verlag, Berlin, 1983.

[20] H. Nakano, Modulared Semi-Ordered Linear Spaces, Maruzen Co., Ltd., Tokyo, 1950. 
[21] N. Samko, Singular integral operators in weighted spaces of continuous functions with oscillating continuity moduli and oscillating weights, In: "The Extended Field of Operator Theory". Operator Theory: Advances and Applications, 171 (2006), 323-347.

[22] I. B. Simonenko, Stability of the weight properties of functions with respect to a singular Cauchy integral, Math Notes, 33 (1983), 208-212.

Departamento de Matemática

Faculdade de Ciências e Tecnologia

Universidade Nova de Lisboa

Quinta da Torre

2829-516 Caparica

Portugal

(E-mail : oyk@fct.unl.pt) 


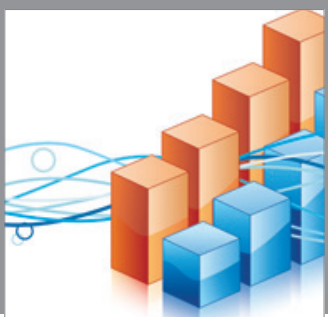

Advances in

Operations Research

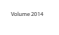

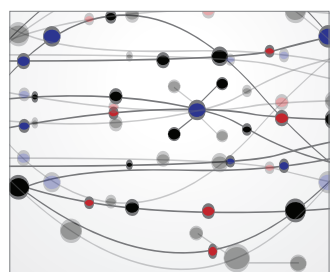

\section{The Scientific} World Journal
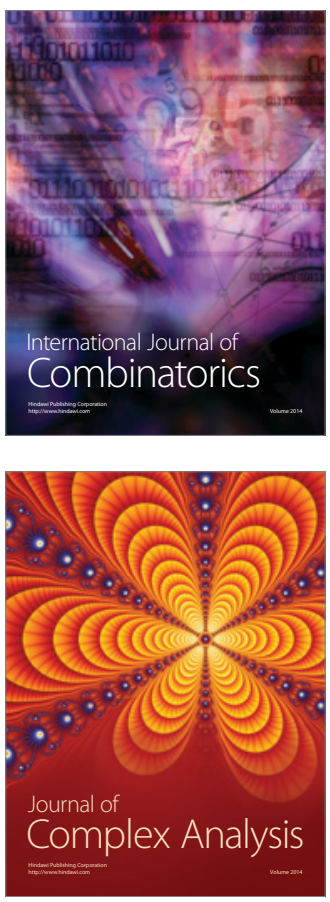

International Journal of

Mathematics and

Mathematical

Sciences
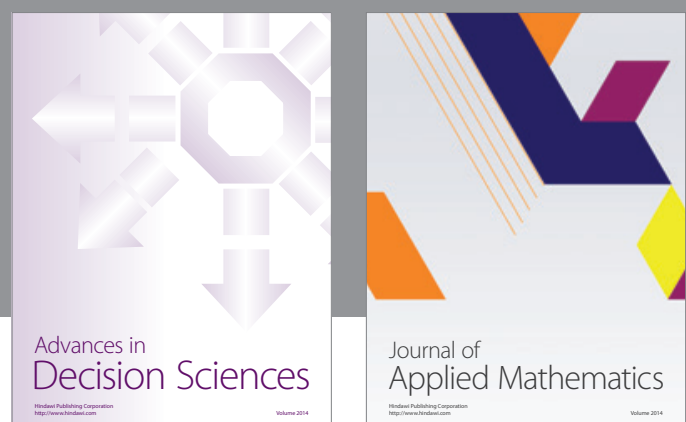

Journal of

Applied Mathematics
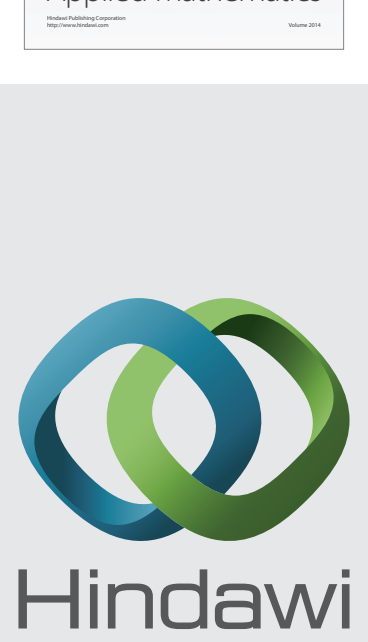

Submit your manuscripts at http://www.hindawi.com
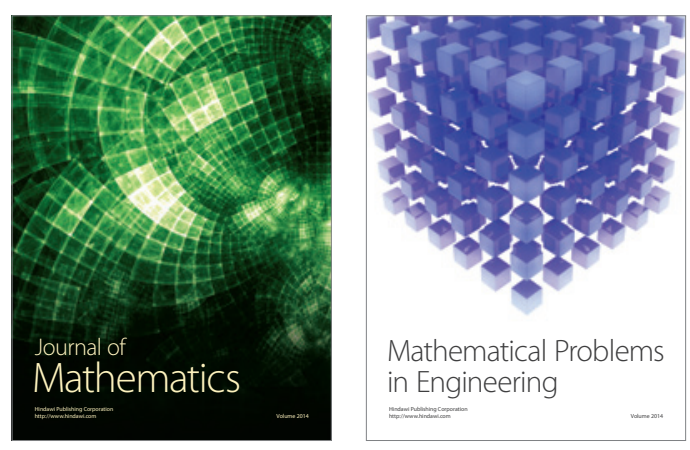

Mathematical Problems in Engineering
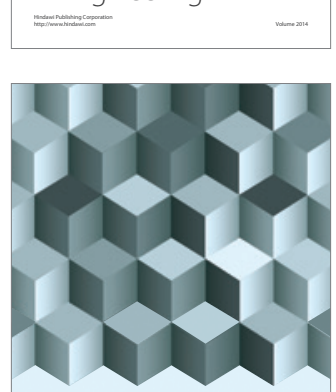

Journal of

Function Spaces
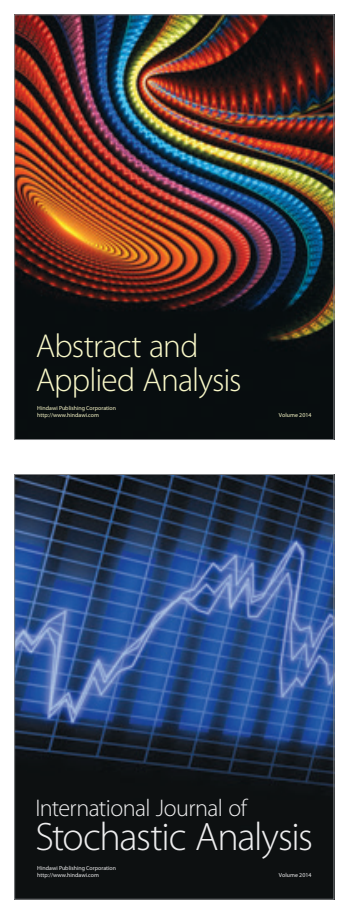

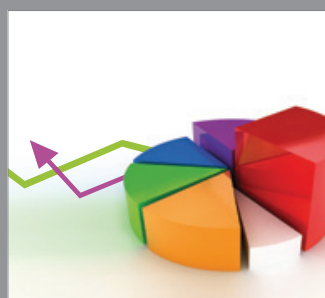

ournal of

Probability and Statistics

Promensencen
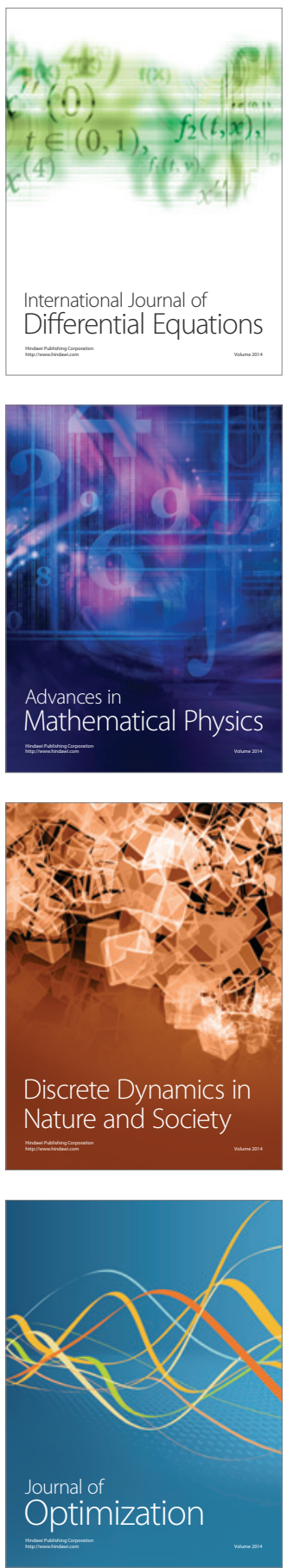